\title{
Human Face Detection Systemin ANew Algorithm
}

\author{
HewaMajeedZangana \\ Department of Computer Science / College of Computer Science and IT / Nawroz University / Kurdistan Region \\ of Iraq
}

\begin{abstract}
Trying to detecting a face from any photo is big problem and got these days a focusing because of it importance, in face recognition system, face detection in one of the basic components. A lot of troubles are there to be solved in order to create a successful face detection algorithm. The skin of face has its properties in color domain and also a texture which may help in the algorithm for detecting faces because of its ability to find skins from photo. Here we are going to create a new algorithm for human face detection depending on skin color tone specially $\mathrm{YCbCr}$ color tone as an approach to slice the photo into parts. In addition, Gray level has been used to detect the area which contains a skin, after that anotherlevel used to erase the area that does not contain skin. The system which proposed applied on many photos and passed with great accuracy of detecting faces and it has a good efficient especially to separate the area that does not contain skin or face from the area which contain face and skin. It has been agreed and approved that the accuracy of the proposed system is $98 \%$ in human face detection.
\end{abstract}

Keywords: face detection, skin color, $\mathrm{YCbCr}$.

\section{Introduction}

The Face of ahuman is carrying a lot of information like emotional and recognizing persons depending on his face. Face revealed in a complex scene and the fragmentation of its background is a precondition for any process verification using faces. Therefore,Powerful detection and diversify sectors is the first step in Build a fool-proof system. In near past face recognition and detection became a point of researchers where many applications and systems has been made depending on this idea of detection like control system to detect suspicious people and video surveillance and tracking faces and so on. The procedures and steps to get these systems of face detection and dividing photo are many and there will be many Barriers like the expression of face and the color of skin and maybe some other objects will be appeared or not related to illumination or may be even the direction of face or the background of the image or the space between object and the camera. The way to find faces from images and localization is called face detection. And it is the important move in face recognition system. Segmenting image is the biggest trouble in image processing. Segmenting face is a substantialmove in the system of recognizing faces, where many techniques areresorting to deal with faces in photos. Thus, segmenting and detecting faces must work successfully in finding the part which contains the face in the image given.

The most point in recognizing face in human is the color of skin and it can be used to detecting faces from images. Even the color of skin is different between faces and many researchers proofed that the difference came from intensity more that chrominance. Various color spaces have been used to extract skin color like RGB, HSV, YCbCr.Methods of face detection can be divided into many features depending on the method itself and the image based method. Many people worked in this field of research like S. PatilKulakarniandH. C. Vijay Lakshmi and they worked on algorithm depending on color space, where they calculate the $\mathrm{YCbCr}$ and $\mathrm{HSI}$ together with Prewitt and canny edge detection approaches. This approach or technique has been used even by other people in the field of research. An algorithm proposed by I. Laurence Aroquiaraj and K. Satheeshwhich named Hit and missed transformed (HMT) depending on Mathematical Morphological operator for face segmentation, this algorithm specified the texture for gram photo to be use as a rule of segmenting face. A.H. Miry and El Abbadi, N.K.,proposed new approach to divide the skin area depending on histogram thresholding. The image which has been input to system filtered to erase many troubles of segmentation like variety in size, color and shapes. In order to enhance the photo a mathematical morphology has been used.

\section{ApproachAnd Proposed System}

In the current paper, we proposed a color image to be input and then to be transform into $\mathrm{YCbCr}$ mode of color space up to the following: 


$$
\left[\begin{array}{l}
y \\
C_{b} \\
C_{r}
\end{array}\right]=\left[\begin{array}{c}
16 \\
128 \\
128
\end{array}\right]+\left[\begin{array}{ccc}
0.299 & 0.587 & 0.114 \\
-0.168 & -0.331 & 0.5 \\
0.5 & -0.418 & -0.081
\end{array}\right] \times\left[\begin{array}{c}
\text { red } \\
\text { green } \\
\text { blue }
\end{array}\right]
$$

Actually here the important component is the $\mathrm{Cr}$, where the process of segmenting face from image will depend on the $\mathrm{Cr}$. Figure 1 represent the process of transformed of image

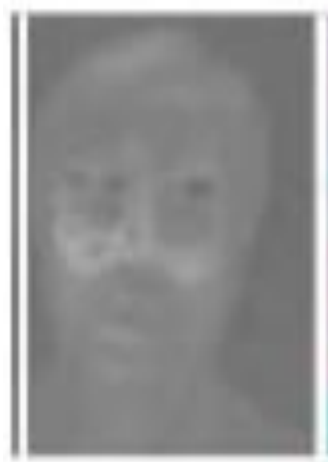

$\mathrm{C}$

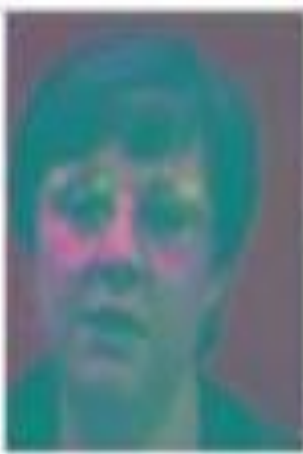

B

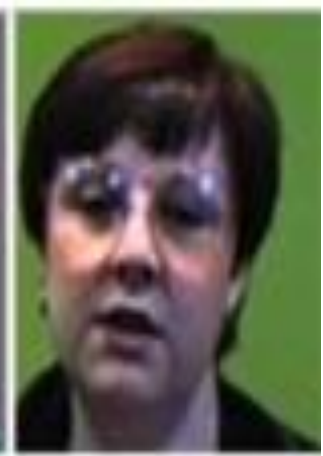

A

Fig. 1:Image in three cases: A. Colored. B. The color space YCbCr. C. Cr Component.

In addition, from the $\mathrm{Cr}$ component a binary image has been generated and this operation complete by taking every value of pixel of image and compare it with the threshold of Cr. Here it can be called detecting skin color tone because of depending on $\mathrm{Cr}$ component, where any value of pixel bigger than the mean of threshold will be skin and it will be colored by white color, where the white color value in image processing is equal to one in value. In the other hand, the pixel that represents the background of the image will be in black color especially where the black color in image processing represented by the value zero. The following is the equation of thresholding:

$$
\text { Threshold }=\frac{1}{w x h} \sum_{i=0}^{w-1} \sum_{j=0}^{h-1} \operatorname{Cr}(i, j)
$$

Where $\mathrm{h}$ and $\mathrm{w}$ representing the height and width of input image, $\mathrm{j}$ and $\mathrm{i}$ indicate the coordinate of the pixel.The following Figure 2 represents the thresholding step

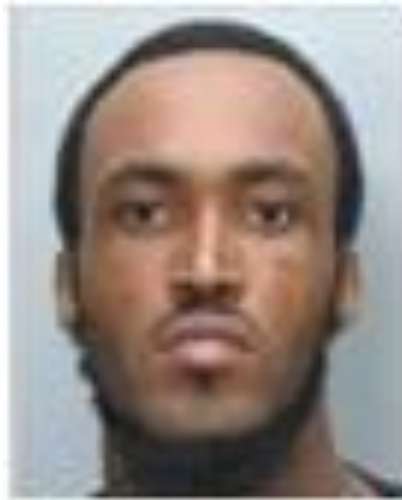

A

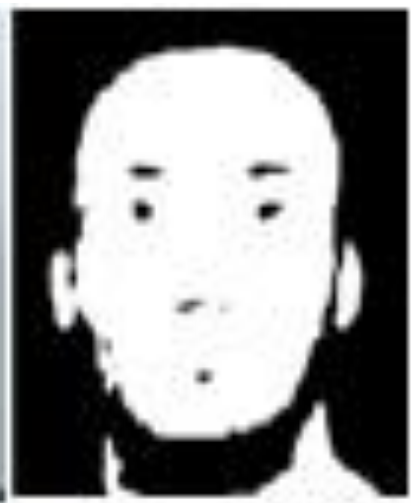

B

Fig. 2: The step of implementing Binary Image: A. Input color image. B. The image in binary case.

After that we will separate the pixels in colored image which had been input into separated area and even edge detection will be applied in order to locate the edges of the input image in order to separate the image into different regions and this will be done by doing the filter of mask as represented in Figure 3 of the input image, wherewe took the image and aplly scanning on it from up to bottom and left to right, after that we will 
apply edge detection by giving one value to the pixel which is matching the mask's center and the other mask's value will be either one or two or three, where the other area of pixels will be represented by zero value.

$$
\left|\begin{array}{lll}
0 & 0 & 0 \\
0 & 1 & 1 \\
0 & 2 & 0
\end{array}\right|
$$

Fig. 3: edge detection mask

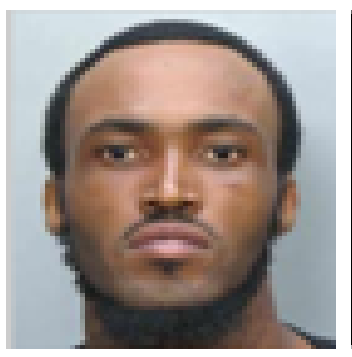

A

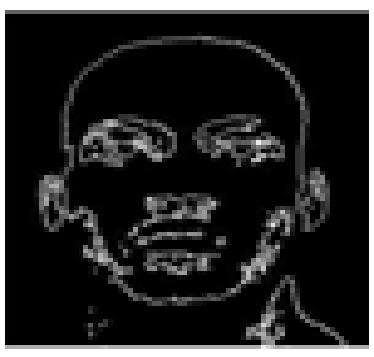

$\mathrm{B}$

Fig. 4: A. color image. B. edged image.

All areas which had been separated and segmented will be numbered from the first area until the last area in the input image. In that matrix a gray level had been used in order to erase the areas which do not contain any face's part. It had been done by the following procedures:

1. The input colored image converted to grayscale.

2. All areas will be divided by giving the all other areas black color and the area which we wanted to divide by giving it the white color. Here if we used more than one image it means we will have more than one white area, it depends on the number of images.

3. Drawing the white area of every single image which come from the second step with gray scale image which had been extracted from input image. Here we got gray color image for the area we assigned and a background of black color.

4. Doing the gray level Co- occurrence of matrix for every area in order to identify the areas which do not contain any part of face and erase it, and this will be done by giving a black color to it.

The proposed equations of this paper as follows:

$$
\begin{aligned}
& \text { Correlation }=\sum_{i=0}^{G-1} \sum_{j=0}^{G-1} \frac{\{i x j\} x P(i, j)-\left\{\mu_{x} * \mu y\right.}{\sigma_{x} * \sigma_{V}} \\
& \text { Inverse Difference Moment }(\mathrm{IDM})=\sum_{i=0}^{G-1} \sum_{j=0}^{G-1} \frac{1}{1+(i-j)^{2}} \quad P(i, j)
\end{aligned}
$$

$$
\text { Angular Second Moment (ASM) }=\sum_{i=0}^{G-1} \sum_{j=0}^{G-1}\{P(i, j)\}^{2}
$$

$$
\text { Contrast }=\sum_{n=0}^{G-1} n^{2} \quad\left\{\sum_{i=0}^{G} \sum_{j=0}^{G} P(i, j) \quad\right\} \cdot|i-j|=n
$$

The operation did not erase the whole non skin area from the photo even it left some areas in the same photo which could be pieces of face area. In order to make the effect of segmentation in the proposed algorithm be increased. Then we are going to concatenate the current photo with the colored original image in order to get the colored area. After that we are going to divide the photo to frames and those frames could be in different sizes and there will be an operation applied in it as follows: 


$$
A_{k} \quad(x, y)=\frac{1}{2^{2 k}} \sum_{i=-2^{k-1}}^{2^{k-1}} \sum_{j=-2^{k-1}}^{-1} f(x+i, y+j)
$$

Where $k=1 \sim 5$ and $f(x+i, y+j)$ is the gray level value of $(x+i, y+j)$.

When $k=1$; a $2 \times 2$ frame is selected to calculate the average pixel value $A 1(x, y)$ of this frame.

When $\mathrm{k}=2, \mathrm{a} 4 \mathrm{x} 4$ frame is used to calculate the average pixel value $\mathrm{A} 2(\mathrm{x}, \mathrm{y})$ of the frame, and so on.

After that it is going to take every value of $\mathrm{k}$ and calculate the average of the gray level which is represent different results from every single pixel to another and the following steps will show the operation as follows:

$$
\begin{aligned}
& E_{k, h}(x, y)=\left|A_{k}\left(x+2^{k-1}, y\right)-A_{k}\left(x-2^{k-1}, y\right)\right| \\
& E_{k, v}(x, y)=\left|A_{k}\left(x, y+2^{k-1}\right)-A_{k}\left(x, y-2^{k-1}\right)\right|
\end{aligned}
$$

Where $(h, v)$ represent the horizontal and vertical directions.

$\mathrm{F}(\mathrm{x}, \mathrm{y})=\max \left\{\mathrm{E}_{\mathrm{k}, \mathrm{i}}(\mathrm{x}, \mathrm{y}), \mathrm{i}=\mathbf{h}\right.$ and $\left.\mathrm{v}, \mathbf{k}=1,2,3,4,5\right\}$

\section{$\mathrm{F}(\mathrm{x}, \mathrm{y})$ represent the coarseness of the pixel $(\mathrm{x}, \mathrm{y})$}

\section{Results}

We have made a large database with many photos in order to test the proposed algorithm on them and they have been shown in fig. 5 , fig. 6 , fig. 7, fig. 8 , fig. 9 , and fig. 10 . The all results come with faces and black backgrounds if the input photo contain face or if there is no face in the input photo the result will be just a black photo. In addition, we have represent some stages of the photo in the operation of applying the proposed algorithm on it starting from the colored photo which has been input until the result photo, like the YCbCr and the $\mathrm{Cr}$ component and the binary photo and finally the result photo which contain the face.
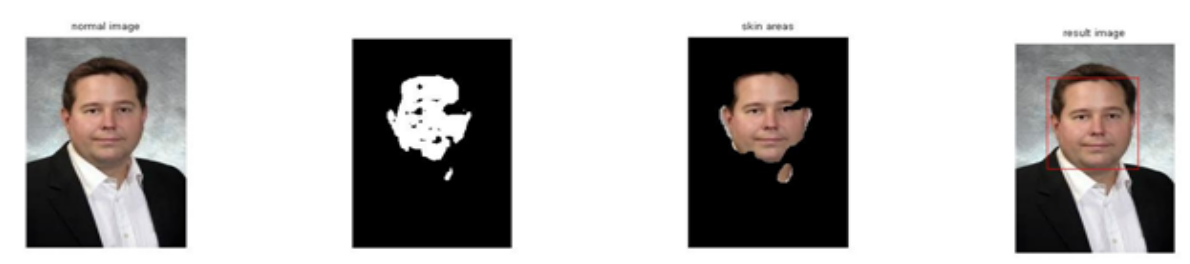

Fig. 5 Test (1) for Proposed Algorithm
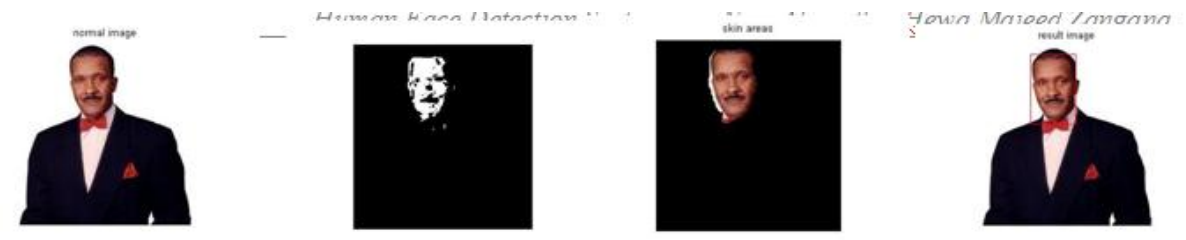

Fig. (6) Test (2) for Proposed Algorithm
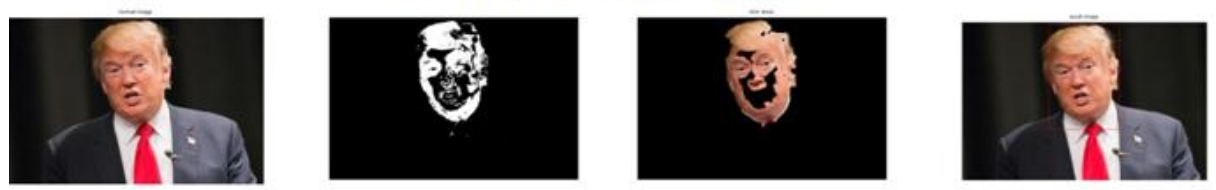

Fig. (7) Test (3) for Proposed Algorithm 

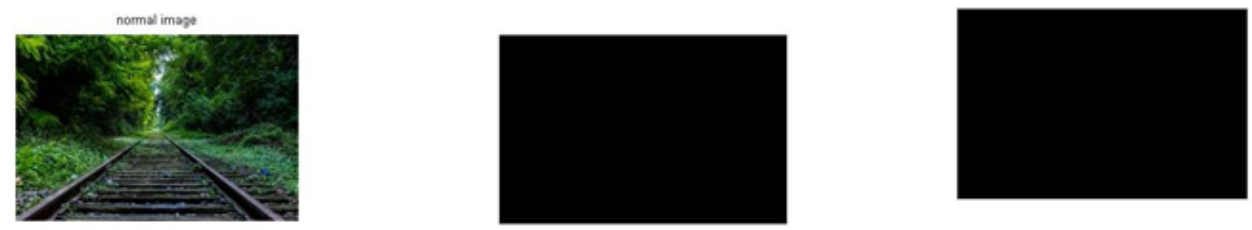

Fig. (8) Test (4) for Proposed Algorithm
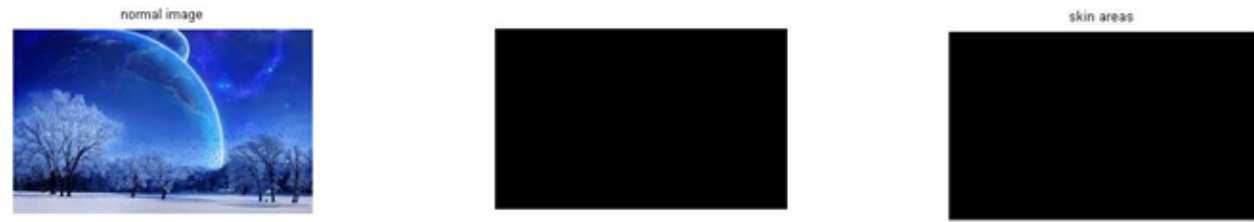

Fig. (9) Test (5) for Proposed Algorithm
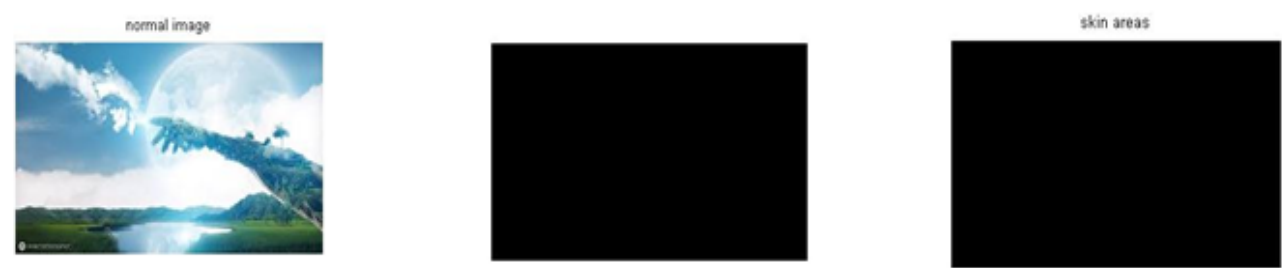

Fib. (10) Test (6) for Proposed Algorithm

In addition, we tested our proposed algorithm on some other photos which contain many faces in order to check the ability of it and how the results will be, the proposed algorithm shows the same results and worked very well and Fig. (11) represents a test of it.
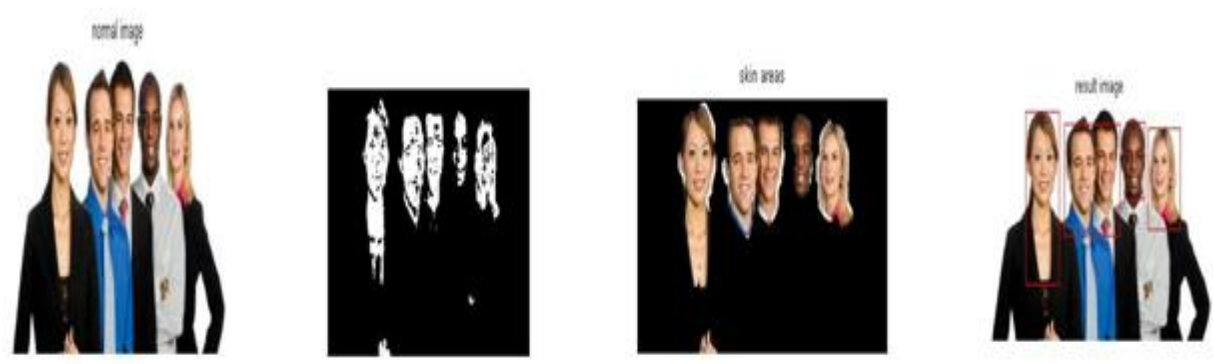

Fig. (11) Test (7) for Proposed Algorithm with a Photo Contains Many Faces

In order to check the accuracy and the performance of proposed system we used criterias called recall and precision like following:

$$
\begin{aligned}
& \text { Precision }=\frac{\text { No.of relevant images retrieved }}{\text { Total no.of images retrieved }} \times 100 \\
& \text { Precision }=\frac{100}{100} \times 100=100 \% \text { for the proposed system } \\
& \text { Recall }=\frac{\text { No.of relevant images retrieved }}{\text { Total no.of relevant images in the database }} \times 100 \\
& \text { Recall }=\frac{99}{100} \times 100=99 \% \text { for the proposed system }
\end{aligned}
$$


We noticed that the time required to detect a face from any photo up to our proposed system took about only two seconds.

\section{Conclusions}

In the current paper we proposed a new algorithm to detect a face from any photo and we depended on the skin color in order to make it be successful algorithm with a high accuracy.

In addition, we divided the photo to some parts, some do not contain skin area and the other containing the skin area of face.The proposed algorithm has been applied on many photos in order to be sure about the accuracy and it is giving the same results with all photos.For future work we recommend to expand the current system to segment and detect the all skin of human body.We compared our proposed algorithm with other exist algorithms and the proposed algorithm gives good results as shown below:

\begin{tabular}{|l|c|c|c|c|}
\hline Criterion & HSI Color Space [9] & YcbcrColor Space [9] & $\begin{array}{c}\text { RGB Color Space } \\
\text { [9] }\end{array}$ & $\begin{array}{c}\text { Proposed } \\
\text { Algorithm }\end{array}$ \\
\hline Accuracy & $73.8 \%$ & $84.61 \%$ & $21.64 \%$ & 98 \\
\hline
\end{tabular}

\section{References}

[1]. Hong, S. and al, e, "Facial feature detection using Geometrical face model: An efficient approach," journal of pattern recognition, Vols. 31, No. 3, pp. 273-282, 1998.

[2]. Leung, C, "Real Time Face Recognition," B. Sc. Project, School of Information Technology and Electrical Engineering. University of Queesland, 2001.

[3]. Sanjay Kr, Singh, D. S. Chauhan, MayankVatsa and Richa Singh, "A Robust Skin Color Based Face Detection Algorithm," Tamkang Journal of Science and Engineering, vol. 6 (4), pp. 227-234, 2003

[4]. Muhammad Tariq Mahmood, "Face Detection by Image Discriminating," $2006 . \quad$ [Online]. Available: http://www.bth.se/fou/cuppsats.nsf/all/6c509ae86a297ca4c12571d300512cac/\$file/DVD009-MasterThesisReport.pdf.

[5]. Michael Padilla and Zihong Fan, "Automatic Face Detection Using Color Based Segmentation and Template / Energy Thresholding," 2003. [Online]. Available: http://www.stanford.edu/class/ee368/Project_03/Project/reports/ee368group16.pdf.

[6]. Rafael C. Gonzalez and Richard E. Woods, Digital Image Processing. 2nded, New Jersey: Prentice-Hall, 2001 , pp. 75-103.

[7]. C. A. Brewer, Color Use Guidelines for Data Representation, Alexandria: American Statistical Association, 1999, pp. P55-56.

[8]. Sangwine, S. J. and Horne, R. E. N, The Color Image Processing Handbook, Chapman \& Hall, 1st Edition, 1998.

[9]. Raghuvanshi, D. S. and Agrawal, D., "Human Face Detection by using Skin Color Segmentation, Face Features and Regions Properties," International Journal of Computer Applications, vol. 38- No.9, 2012.

[10]. HewaMajeedZangana, ImadFakhri Al-Shaikhli, A New Algorithm for Human Face Detection Using Skin Color Tone, IOSR Journal of Computer Engineering, Volume 11, Issue 6, pp 31-38, 2013 